\title{
Cambio de diagnóstico y de categoría diagnóstica en pacientes con artritis idiopática juvenil
}

\author{
Change of diagnosis and diagnostic category in patients with juvenile idiopathic \\ arthritis
}

\author{
Alexis L. Strickler ${ }^{\mathrm{a}}$, Daniela Cifuentes ${ }^{\mathrm{b}}$, Krasna Mihovilovic ${ }^{\mathrm{b}}$, \\ Francisca Vergara ${ }^{\mathrm{b}}$, Mónica Grez ${ }^{\mathrm{c}}$, Viviana Rivera ${ }^{\mathrm{d}}$
}

\author{
aEscuela de Medicina, Departamento de Pediatría, Universidad San Sebastián sede Patagonia, Hospital Dr. Eduardo Schütz Schroeder. Puerto \\ Montt. Chile

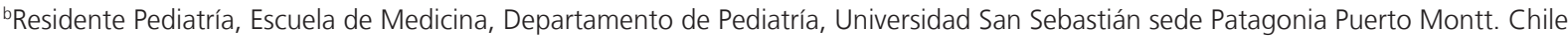 \\ 'Enfermera Pediátrica, Centro de atención abierta, Hospital Dr. Eduardo Schütz Schroeder. Puerto Montt. Chile \\ dEscuela de Medicina, Departamento de Salud Pública, Universidad San Sebastián sede Patagonia, Puerto Montt, Chile.
}

Recibido: 12 de agosto de 2019; Aceptado: 27 de abril de 2020

¿Qué se sabe del tema que trata este estudio?

La artritis idiopática juvenil (AIJ) de inicio antes de los 16 años de edad, puede evolucionar hasta la adultez con episodios de reagudización y secuelas, aún con tratamiento oportuno y efectivo, requiriendo transición organizada, control y ajustes de terapia periódicos.
¿Qué aporta este estudio a lo ya conocido?

La AIJ puede asociarse a otras patologías autoinmunes. Los factores de riesgo pueden diferir en poblaciones rurales vs urbanas, entre individuos y lugares con distinto acceso a salud especializada. Es indispensable auditar frecuentemente el manejo pediátrico y post transición.

\section{Resumen}

$\mathrm{Al}$ menos 50\% de los pacientes pediátricos portadores de artritis idiopática juvenil (AIJ) continuará control en reumatología adulto. La clasificación de la Liga Internacional de Asociaciones de Reumatología (ILAR) vigente, actualmente en revisión, difiere de la clasificación de las artritis inflamatorias del adulto. Se ha reportado cambios de categoría en 10,8\% de los pacientes durante el seguimiento. Objetivo: Analizar los pacientes con AIJ seguidos al menos 7 años para objetivar cambios de diagnóstico en la transición, e identificar factores de mal pronóstico funcional. Pacientes y Método: Estudio retrospectivo en base a registros clínicos. Se incluyó a la totalidad de los pacientes con AIJ controlados en policlínico pediátrico del Hospital de Puerto Montt entre el año 2005 y 2017, que cumplieron siete o más años de seguimiento. Se realizó análisis descriptivo en base a variables clínicas: categoría diagnóstica, tiempo de evolución al diagnóstico, actividad clínica y serológica, y tiempo de evolución
Palabras clave: Artritis idiopática juvenil; Pediatría; Transición cuidado adulto; Reumatología

Correspondencia:

Alexis L. Strickler

alexisstrickler@gmail.com 
al inicio de la terapia farmacológica. Resultados: Se evaluaron 18 pacientes, 3 Oligo-articular (OA) persistente, 1 OA extendida, 4 Poli-articular (PA) factor reumatoide (FR) negativo, 4 PA FR positivo, 5 Sistémicas, 1 Psoriática, todos con seguimiento mayor a 7 años. Once de 18 niños fueron transferidos a adultos. Tres de 11 cambiaron de diagnóstico a Artritis Reumatoide (AR) más otra enfermedad autoinmune: Síndrome de Sjögren + Lupus eritematoso sistémico, Púrpura trombocitopénico inmune, Enfermedad autoinmune no clasificada y cinco de 11 niños de categoría ILAR: OA a Artritis reumatoide juvenil, OA extendida a PA FR negativo, 3 Sistémicas a PA FR negativo. Edad de inicio, formas poli-articulares, retrasos en diagnóstico y comienzo de terapia se asociaron a secuelas e inflamación persistente. Conclusiones: Ocho de once pacientes transferidos cambiaron denominación diagnóstica y/o presentaron otras enfermedades autoinmunes. Algunos factores de mal pronóstico deben mejorar.

\section{Abstract}

At least $50 \%$ of pediatric patients with Juvenile Idiopathic Arthritis (JIA) will require continued follow-up in adult rheumatology. The present International League of Associations for Rheumatology (ILAR) classification, currently under revision, differs from its classification of inflammatory arthritis in adults. Category changes have been reported in $10.8 \%$ of patients during follow-up. Objective: To analyze JIA patients in follow-up for at least 7 years to detect diagnosis changes during transition to adult care, identifying factors of poor functional prognosis. Patients and Method: Retrospective study based on medical records of JIA patients seen at the pediatric polyclinic of the Puerto Montt Hospital between 2005 and 2017, who were monitored for at least 7 years. Descriptive analysis was performed according to clinical variables: diagnostic category, evolution before diagnosis, clinical and serological activity, and evolution before starting drug therapy. Results: We evaluated 18 patients, corresponding to 3 patients with persistent oligoarticular arthritis (OA), 1 with extended OA, 4 with polyarticular arthritis (PA) rheumatoid factor (RF) negative, 4 with PA RF positive, 5 with systemic JIA, and 1 with psoriatic arthritis, all have had follow-up more than 7 years. 11 out of 18 patients transitioned to adult care. Three out of 11 patients changed diagnosis to Rheumatoid Arthritis (RA) plus another autoimmune disease such as Sjögren's Syndrome + Systemic Lupus Erythematosus, Immune thrombocytopenia, or unclassified autoimmune disease, and 5 out of 11 children changed ILAR category from OA to Juvenile Rheumatoid Arthritis, extended OA to PA RF negative, and 3 from Systemic arthritis to PA RF negative. Age of onset, polyarticular forms, delay in diagnosis, and the start of therapy were associated with sequelae and persistent inflammation. Conclusions: Eight of the eleven JIA patients who transitioned to adult care changed their diagnosis or presented other autoimmune diseases. Some factors of poor prognosis must improve.

\section{Keywords:}

Juvenile Idiopathic

Arthritis;

Pediatrics;

Transition Adult Care; Rheumatology

\section{Introducción}

La artritis idiopática juvenil (AIJ) es la patología reumatológica pediátrica más frecuente. Se caracteriza por inflamación articular de más de 6 semanas, de causa desconocida, e inicio antes de los 16 años de edad. Incluye 7 categorías mutuamente excluyentes: OA persistente o extendida, PA FR positivo, PA FR negativo, psoriática, asociada a entesitis, sistémica e indiferenciada, definidas en los primeros 6 meses de evolución, en base a parámetros clínicos: número de articulaciones afectadas, presencia de anticuerpos específicos, compromiso de entesis, asociación con Psoriasis e inflamación sistémica ${ }^{1-3}$, que se correlacionan parcialmente con el pronóstico articular y se especula que tendrían distinta etiopatogenia y base genética ${ }^{4}$. La incidencia reportada varía entre 2-20 casos por 100.000 niños, con amplia dispersión geográfica ${ }^{1-4}$. En Chile se estima en 10 casos por 100.000 menores de 16 años. La clasificación ILAR 2001 vigente, está actualmente en revisión y proceso de cambio, tanto del número de categorías y los elementos clínicos que las definen, como en relación con las artropatías autoinmunes del adulto: AR seropositiva o seronegativa, enfermedad de Still y espondiloartropatías entre las que se incluye la artropatía psoriática.

El objetivo del trabajo es presentar los datos relacionados con la clasificación inicial de pacientes con AIJ seguidos por más de 7 años, detectar el cambio en la denominación diagnóstica posterior a la transferencia a reumatología adultos y analizar los factores de persistencia de inflamación y secuelas de nuestra cohorte, lo que contribuirá a diseñar estrategias de mejoría de nuestros procesos clínicos y del proceso de transición. 


\section{Pacientes y Método}

\section{Diseño}

Estudio de tipo retrospectivo en base a registros clínicos. Se incluyó a la totalidad de los pacientes con AIJ controlados en policlínico pediátrico del Hospital de Puerto Montt (HPM) entre el año 2005 y 2017, que cumplieron siete o más años de seguimiento, 18 en total.

\section{Variables analizadas}

Se realizó análisis descriptivo en base a variables clínicas: categoría diagnóstica, tiempo de evolución al diagnóstico, actividad clínica y serológica, y tiempo de evolución al inicio de la terapia farmacológica.

\section{Análisis estadístico}

La variable cualitativa (categoría diagnóstica) se expresó en cifras absolutas. La variable cuantitativa (tiempo de evolución al inicio de tratamiento) se expresó con su media y desviación estándar, los grupos fueron comparados mediante diferencia de promedios aplicándose como prueba estadística t test. Se consideró significancia con valor de $\mathrm{p}<0,05$.

\section{Ética}

Se aplicó previamente consentimiento informado y aprobación de comité de ética científica acreditado

\section{Resultados}

Dieciocho de 42 pacientes diagnosticados entre 1998 y 2011, controlados en HPM entre 2005 a 2017 tuvieron seguimiento en el HPM de 7 años y más (rango 8-14 años). Aplicando la clasificación ILAR se distribuyeron en: 3 OA, 1 OA extendida, 4 PA FR negativo, 4 PA FR positivo, 5 Sistémicas, y 1 Psoriática. No hubo pacientes con AIJ indiferenciada. Solo una de las tres pacientes con la forma OA presentó uveítis.

Once de 18 pacientes fueron transferidos a adultos. Ocho de 11 pacientes en control con reumatólogo/a adulto han cambiado de denominación, 3 de ellos a AR más otra patología autoinmune y 5 de categoría dentro de la clasificación ILAR (tabla 1).

Cuatro de 7 pacientes no transferidos, alcanzaron la remisión total previo a la edad de transición. 2 menores de 15 años, ambos en remisión clínica y serológica con Tocilizumab (TCZ) luego de falta de respuesta a 2 anti-TNF previos: Etanercept y Adalimumab y 1 de 19 años que ha continuado en control con inmunoreumatóloga pediátrica del centro de referencia por negativa de los padres a transición y aceptación del médico tratante.

En relación a la clasificación inicial, el estado clínico actual es: 5/18 se encuentran en remisión sin tratamiento y sin secuelas, 3 OA, 1 psoriática y 1 sistémica pura. 1/18 diagnosticada como sistémica asociada a enfermedad autoinmune no clasificada, se encuentra

Tabla 1. Distribución según diagnóstico

\begin{tabular}{|c|c|c|c|}
\hline \multicolumn{2}{|l|}{ Diagnóstico inicial } & \multicolumn{2}{|l|}{ Diagnóstico a los 7 años } \\
\hline Categoría & $\mathrm{n}$ & Categoría & $\mathrm{n}$ \\
\hline \multirow[t]{2}{*}{ AlJ OA persistente } & 3 & AIJ OA & 2 \\
\hline & & ARJ & 1 \\
\hline AlJ OA extendida & 1 & AlJ PA FR negativo & 1 \\
\hline \multirow[t]{2}{*}{ AIJ PA FR positivo } & 4 & AlJ PA FR positivo & 3 \\
\hline & & $A R+$ Síndrome de Sjodren + LES & 1 \\
\hline \multirow[t]{2}{*}{ AlJ PA FR negativo } & 4 & AlJ PA FR negativo & 3 \\
\hline & & $A R+P T I$ & 1 \\
\hline \multirow[t]{3}{*}{ AlJ Sistémica } & 5 & AlJ Sistémica & 1 \\
\hline & & AlJ PA FR negativo & 3 \\
\hline & & AR + Enfermedad autoinmune no especificada & 1 \\
\hline AlJ Psoriática & 1 & AlJ Psoriática & 1 \\
\hline AlJ Indiferenciada & 0 & & \\
\hline Total & 18 & Total & 18 \\
\hline
\end{tabular}

AlJ: Artritis idiopática juvenil, OA: oligoarticular, PA: poliarticular, FR: factorreumatoideo, ARJ: Artritis reumatoide juvenil, AR: Artritis reumatoide, LES: Lupus eritematoso sistémico, PTI: púrpura trombocitopénicoinmune. 
en remisión sin tratamiento pero con secuelas. 3/18 sistémicas con afectación PA, están en remisión con tratamiento y daño articular crónico. 3/18 PA FR negativo en remisión con tratamiento y sin secuelas a la fecha. 6/18; 3 PA FR positivo una de ellas con Síndrome de Sjödren y 3 PA FR negativo, una de ellas con PTI, se encuentran activas con tratamiento. 7/18 tienen Artrosis (tabla 2). Los factores asociados a actividad y presencia de secuelas fueron: las formas poli-articulares y tiempo excesivo de evolución, mayor a 3 meses, previo al inicio de terapia con metotrexato (MTX) y terapia biológica, sin embargo estas diferencias, considerando el inicio tardío del tratamiento y dado el escaso número de pacientes no resultaron ser estadísticamente significativas (tabla 3). En el caso de la paciente con la forma OA anticuerpo antinuclear (ANA) positivo que desarrolló uveítis y sus complicaciones, incidieron la edad de comienzo menor a 2 años, retraso en el diagnóstico e inicio de terapia modificadora de la enfermedad (FARME), e inicio y continuidad de terapia biológica, previo al programa GES, y la falta de respuesta a 3 anti-TNF; Etanercept, Adalimumab e Infliximab y a un modulador selectivo de co-estimulación de Linfocitos T, Abatacept.

Tabla 2. Condición actual 18 pacientes a siete años de evolución

\begin{tabular}{llll}
\hline Remisión & Sin tratamiento & Sin secuelas & 5 \\
& & Con secuelas & 1 \\
& Con tratamiento & Sin secuelas & 3 \\
& & Con secuelas & 3 \\
Activa & Sin secuelas & & 3 \\
& Con secuelas & & 3 \\
Total & & 18 \\
\hline
\end{tabular}

\section{Discusión}

Conocer la evolución longitudinal de nuestros pacientes con AIJ y los factores de riesgo de secuelas, respecto de los subtipos vigentes, incluyendo el diagnóstico asignado luego de la transición a reumatólogos adultos, es importante para mejorar nuestros procesos de atención, junto con incorporar sistemáticamente los avances en el conocimiento, incluyendo terapias más precoces y efectivas. Previo a la guía clínica y programa Garantías Explícitas en Salud (GES), se hacía el diagnóstico genérico de artritis reumatoide juvenil (ARJ), había pocos médicos especialistas, concentrados en regiones V, VI, VII, VIII, IX y metropolitana del país ${ }^{5}$ y algunos pacientes probablemente eran tratados demasiado tarde, inadecuadamente o no acorde con las opciones más modernas de terapia, situación descrita en otros países ${ }^{6}$. En noviembre de 1998 la Federal Drug Administration (FDA) aprobó el uso de Etanercept para AR y en mayo de 1999 para AIJ PA. Posteriormente han aparecido otros medicamentos biológicos, con el potencial de mejoría y/o remisión total o parcial de la enfermedad ${ }^{6-10}$. En Chile desde el año 2010, los pacientes con AIJ de la salud pública y priva$\mathrm{da}$, son ingresados, a la patología GES $\mathrm{N}^{\circ} 63 \mathrm{AIJ}$, que adopta la denominación de AIJ, la clasificación ILAR, guía clínica y medicamentos biológicos, guía que es actualizada por reumatólogos pediátricos el año $2014^{11}$. En base a esa clasificación, vigente aún, ya habíamos demostrado que en nuestra población total de AIJ reportada el año 2018, el fenotipo PA FR negativo, fue el más frecuente $(33 \%)$ y la categoría PA FR positivo alcanzó el $17 \%{ }^{12}$, adiferencia de otras regiones de Chile, y países occidentales, donde predomina la forma $\mathrm{OA}^{11,13}$, pero similar a otros países: Suecia, Sudáfrica, Alemania, India, Nueva Zelandiaa ${ }^{7,14}$. Sin embargo es posible que esta diferencia respecto a datos nacionales se deba a subdiagnóstico de las formas OA. Nuestra incidencia calculada entre los años 2005-2015 oscila en-

Tabla 3. Condición actual a 7 años, según tiempo de evolución al inicio de terapia farmacológica

\begin{tabular}{|c|c|c|c|c|c|}
\hline Inicio Metotrexato: & 14 meses (DS $\pm 12 \mathrm{~m}$ ) & $p=0,3$ & Inicio Metotrexato: & 22 meses $(\mathrm{DS} \pm 28 \mathrm{~m})$ & $p=0,3$ \\
\hline Inicio Biológicos: & 5,8 años (DS $\pm 3,3$ a) & $p=0,8$ & Inicio Biológicos: & 5,4 años (DS $\pm 3,9$ a) & $p=0,8$ \\
\hline \multicolumn{3}{|l|}{ REMISIÓN ( $n=12$ ) } & \multicolumn{3}{|l|}{ ACTIVIDAD $(n=6)$} \\
\hline Inicio Metotrexato: & 11 meses $(D S \pm 11 \mathrm{~m})$ & $p=0,3$ & Inicio Metotrexato: & 22 meses $(D S \pm 24 \mathrm{~m})$ & $p=0,3$ \\
\hline Inicio Biológicos: & 4 años (DS $\pm 2,1 \mathrm{a})$ & $p=0,4$ & Inicio Biológicos: & 7 años (DS $\pm 3,8$ a) & $p=0,4$ \\
\hline \multicolumn{3}{|c|}{ SIN SECUELAS $(n=11)$} & \multicolumn{3}{|c|}{ CON SECUELAS $(n=7)$} \\
\hline
\end{tabular}

DS: desviación estándar. 
tre $0-4,9$ casos año por 100.000 niños, lo que también sugiere subdiagnóstico en nuestra región. Sería importante conocer la incidencia y prevalencia nacional actuales, junto con los fenotipos más frecuentes en distintas regiones del país, los que podrían no coincidir con los fenotipos previamente reportados en base preferentemente a población de la Región Metropolita$\mathrm{na}^{11,13}$. Nordal et al 2011, en un seguimiento a largo plazo de pacientes con AIJ ha reportado cambios de la categoría inicial en $10,8 \%$ de su cohorte y persistencia de actividad inflamatoria ${ }^{15}$, lo que nos motivó a estudiar nuestra cohorte. La dificultad para lograr remisión se ha asociado entre otros factores, al subtipo de AIJ, siendo las formas PA FR positivo o negativo, artritis asociada a entesitis (ERA por la sigla en inglés) y sistémica con compromiso PA las que persisten con inflamación. De ellas entre el $50-60 \%$ son transferidos a reumatología adultos ${ }^{15-17}$. Se ha descrito también que la evolución clínica de los pacientes con AIJ aún dentro del mismo subtipo es variable e impredecible ${ }^{15}$. Las formas OA son las que presentan mejor evolución y tasa de remisión más alta ${ }^{15-18}$ lo que podemos apreciar en este grupo de pacientes. Hace excepción la paciente de inicio temprano, 1 año y 6 meses, ANA positivo, diagnóstico tardío por ausencia de especialista, desarrollo precoz de uveítis y acceso a terapia biológica tardía e irregular al comienzo cuando no existía el programa GES, que condicionaron mal pronóstico funcional articular y ocular. En relación a pronóstico funcional, Wallace et al 2012 y Albers et al 2010 han propuesto como objetivo ideal del manejo de AIJ, alcanzar la remisión o al menos inflamación mínima, antes de los dos primeros años de la enfermedad, evitando el dolor crónico y disminuyendo la discapacidad funcional ${ }^{19,20}$, objetivos que no se han cumplido en esta cohorte. Albers et al 2010 han señalado que el factor más significativo para predecir actividad de la enfermedad en los años posteriores es la cantidad de tiempo con enfermedad activa durante los primeros 2 años de la enfermedad, siendo predictores de reagudización, PCR o VHS elevadas, curso poli-articular y falta de respuesta a metotrexato ${ }^{20}$. Antes del año 2010, como en otras casuísticas reportadas, nuestros pacientes recibieron distintos esquemas de tratamiento y el retraso tanto en el diagnóstico como en el inicio del tratamiento objetivados en los resultados (tabla 3), derivados de conocimiento insuficiente de la enfermedad a nivel pediátrico, ausencia de guía clínica con acceso a terapias efectivas y falta de especialista reumatólogo o inmunólogo pediátricos a nivel local, condicionaron una alta tasa de secuelas. El acceso a los medicamentos biológicos no ha sido uniforme en distintos países, como tampoco la forma de uso $^{6}$, lo que también ha ocurrido en Chile, especialmente a nivel regional. Algunos pacientes han tenido acceso a terapia efectiva relativamente oportuna, in- cluyendo medicamentos biológicos desde el año 2010 y otros, no los han recibido o se han iniciado tardíamente (tabla 3). Otros factores no relacionados con los aspectos biológicos de la enfermedad detectados en nuestra población son: Escaso conocimiento de la patología a nivel primario de salud, retraso en el diagnóstico por razones demográficas y de organización; accesibilidad a consulta especializada, retraso en exámenes inmuno-reumatológicos e imagenológicos como ecografía, retardo en la administración de inmunizaciones previo al inicio de FARMES, disponibilidad y oportunidad de FARMES incluido biológicos, acceso, calidad y persistencia de rehabilitación, todos factores precisados en el reporte del año $2018^{12}$ que son modificables con educación y planificación. Nuestra casuística en relación a las publicaciones internacionales es pequeña, menor aún el número de pacientes que cumple la edad de transición y vive en una zona geográfica determinada, por lo que no podemos extraer resultados de significación estadística, sin embargo el seguimiento de nuestros pacientes demuestra que un número significativo llega con inflamación, tratamiento y/o secuelas a la edad de transferencia a reumatología de adultos.A pesar de amplia experiencia internacional en programas estructurados de transición que abordan éste y otros temas particulares de la adolescencia como prevención de embarazo, consumo de tabaco, alcohol y otras sustancias, ejercicio y deporte, cambios psicológicos normales o anormales y relación de pares ${ }^{21-27}$, en nuestro conocimiento no se ha reportado en Chile los resultados de un programa de transición en AIJ y en nuestra región no hemos implementado aún un programa formal de transición. Los únicos criterios utilizados en nuestro hospital para transferencia han sido la persistencia de síntomas o ausencia de remisión, edad mayor de 17 años y la falta de profesionales reumatólogo o inmunólogo o pediatra capacitado en reumatología en algunos períodos. El traslado se ha efectuado con interconsulta, sin proceso de conocimiento previo de pacientes y reumatólogos de adultos, lo que claramente contrasta con las recomendaciones de EULAR publicadas en Foster et al $2016^{26} \mathrm{y}$ actualizadas en Conti et al $2018^{27}$. Algunos reumatólogos/as de adultos no han atendido previamente pacientes con AIJ, lo que ocurre también en otros países, por lo que algunos pacientes son diagnosticados, inmediatamente después del traspaso, de acuerdo a la clasificación de artropatías inflamatorias del adulto principalmente $\mathrm{AR}^{28,29}$. Esta situación más la evolución hacia otras enfermedades autoinmunes ha ocasionado cambios en la denominación diagnóstica de $08 / 11$ pacientes transferidos, algunos esperables como la aparición de otras enfermedades autoinmunes y otros que muestran la falta de un programa de transición estructurado que considere la atención conjunta de pacientes durante el período de 
transición, manteniendo la clasificación AIJ luego del traslado a adultos como se reporta en las experiencias de transición publicadas, lo que en nuestro país significa además mantener los beneficios de acceso, oportunidad y terapia de una patología garantizada por ley. La canasta de medicamentos AIJ del programa GES ha incorporado medicamentos biológicos en forma progresiva: etanercept año 2010, adalimumab e infliximab año 2012 y el año 2019 tocilizumab para las formas sistémica y PA que no responden a tratamiento con metotrexato y antiTNF. Desde el año 2007 (última actualización MINSAL 2014) la AR es la patología Nº 52 del sistema GES. Las guías clínicas nacionales de AIJ y AR difieren en el tipo de pacientes incluidos, ya que AR solo considera formas PA con FR o anticuerpos citrulinados (ACPA) positivos fuerte, débil o negativos, y no incluye las formas $\mathrm{OA}$, sistémicas, psoriáticas y asociadas a entesitis, consideradas dentro del diagnóstico diferencial, categorías que sí están incluidas en la clasificación ILAR de AIJ. También difieren en los esquemas de tratamiento utilizados, especialmente FARMES, el momento y tipo de biológicos a utilizar (guías 52 y 63 GES MINSAL). Las artropatías psoriáticas y espondiloartropatías del adulto no están incluidos en GES, sin embargo se puede acceder a biológicos mediante ley de medicamentos de alto costo. En consonancia con las diferentes guías de terapia de las patologías inflamatorias articulares crónicas del adulto y del menor de 17 años, se ha detectado diferencias en el uso de medicamentos entre las poblaciones de AIJ PA FR positivo o negativo y AR, AIJ sistémica vs enfermedad de Still , AIJ psoriática y artritis psoriática del adulto, AIJ asociada a entesitis y espondiloartropatías, con mayor prevalencia del uso de inhibidores de TNF, IL-1 e IL-6 en población pediátrica y excepcional uso de hidroxicloroquina y azulfidina en niños ${ }^{6,28,29}$, lo que se refleja en la atención pediátrica de nuestros pacientes y en la transición. Así, es importante mantener la denominación de AIJ y los fenotipos correspondientes luego de la transición, de modo de que los pacientes accedan a terapias aseguradas por programa GES AIJ durante toda su evolución. Distintos investigadores enfatizan la falta de guía consensuada de tratamiento para AIJ del adulto ${ }^{26-29}$. Publicaciones recientes analizan críticamente la clasificación AIJ de ILAR y la clasificación de artropatías del adulto, señalando que es necesario revisar y actualizar dicha clasificación tomando en consideración los avances en el conocimiento genético y fisiopatológico, así como los estudios de seguimiento clínico de los pacientes AIJ. Estos estudios han mostrado una gran concordancia entre AIJ PA FR positiva y AR seropositiva por lo cual constituirían la misma artropatía crónica autoinmune manifestada desde la infancia ${ }^{30,31}$. Adicionalmente se han reportado similitudes en síntomas y signos sistémicos de inflamación, ausencia de auto-anticuerpos entre AIJ sistémica y enfermedad de Still por lo que se ha postulado que ambas serían también la misma enfermedad inflamatoria en distinta etapa del desarrollo ${ }^{32,33}$. Además se ha analizado los factores comunes, compromiso de entesis y asociación con HLA-B27, entre las ERA versus espondiloartropatías, y las diferencias respecto del predominio de la forma axial o periférica, siendo la última más frecuente en edad pediátrica ${ }^{34}$. Algunos investigadores sostienen que la AIJ OA ANA positivo sería un fenotipo exclusivo o preferente de la niñez ${ }^{35}$, aunque Nigrovic et al 2018 manifiestan dudas al respecto ${ }^{36}$. Se ha señalado que la presencia de psoriasis en el paciente o sus familiares directos como único elemento, en ausencia de dactilitis o compromiso entésico, no sería una herramienta útil para configurar el fenotipo de artritis psoriática dentro de la clasificación ILAR de $\mathrm{AIJ}^{37}$ y publicaciones recientes sobre artritis psoriática, que incluyen la artritis psoriática infantil, confirman la relación con las espondiloartropatías del adulto ${ }^{38}$. Por estas razones se ha propuesto disminuir dentro de la clasificación ILAR de 7 a 4 subtipos $^{36,39}$, e incluso a 3 categorías $^{40}$. Ello permitiría uniformar criterios de terapia y seguimiento desde la edad pediátrica en adelante, disminuir los cambios de diagnóstico y probablemente optimizar el tratamiento y seguimiento. Por ahora debe mantenerse la clasificación AIJ actual, y transferir nuestros pacientes a reumatología adulto con la denominación correspondiente, en las mejores condiciones posibles en cuanto a persistencia de inflamación y secuelas, en un proceso de transición estructurado de acuerdo a las recomendaciones de EULAR ${ }^{26}$.

Finalmente parece fundamental, la presencia y distribución equitativa de especialistas en reumatología pediátrica en las distintas regiones del país, la difusión del conocimiento de estas enfermedades a nivel primario de salud, la uniformidad en las pautas de tratamiento que incorporen oportunamente los avances en el manejo no farmacológico y farmacológico de estas patologías, la evaluación periódica de la actividad de la inflamación con las herramientas disponibles: Escala visual análoga de dolor, inflamación y sensación de bienestar del paciente, familiar y médico, puntuación de actividad de la enfermedad articular (JADAS por la sigla en inglés) o puntuación de actividad de enfermedad (DAS por la sigla en inglés), Cuestionarios específicos de calidad de vida (CHAQ), VHS, PCR y un enfoque dirigido al paciente desde la niñez en adelante, que considere los factores personales de riesgo para la indicación y cambio precoz de terapias disponibles e instaurar el programa de transición en conjunto con reumatólogos de adultos de acuerdo a los criterios de EULAR,que considere la atención conjunta por un período determinado, de modo de facilitar la continuidad de la atención en esta población especial de pacientes. 
En conclusión, en nuestra casuística hubo cambios de diagnóstico y categoría en consonancia con lo descrito internacionalmente y el proceso de transición ha sido inadecuado. Visualizar los factores de riesgo de secuelas en nuestra población, efectuando periódicamente auditoría del manejo realizado, permitirá planificar las acciones para evitarlos o minimizarlos, transferir a los adolescentes con mejor calidad de vida y organizar una transición adecuada y completa a reumatología adultos.

\section{Responsabilidades Éticas}

Protección de personas y animales: Los autores declaran que los procedimientos seguidos se conformaron a las normas éticas del comité de experimentación humana responsable y de acuerdo con la Asociación Médica Mundial y la Declaración de Helsinki.

Confidencialidad de los datos: Los autores declaran que han seguido los protocolos de su centro de trabajo sobre la publicación de datos de pacientes.

\section{Derecho a la privacidad y consentimiento informa-} do: Los autores han obtenido el consentimiento informado de los pacientes y/o sujetos referidos en el artículo. Este documento obra en poder del autor de correspondencia.

\section{Conflicto de intereses}

Los autores declaran no tener conflicto de intereses.

\section{Agradecimientos}

Los autores agradecen al Dr. José Antonio Vergara, epidemiólogo de la SEREMI de salud de la X región de Los Lagos, por el cálculo de incidencia de AIJ en nuestra población y al Sr. Felipe Campos por el apoyo en la búsqueda bibliográfica.

\section{Referencias}

1. Petty RE, Southwood TR, Manners P, et al. International league of associations for rheumatology classification of juvenile idiopathic arthritis: second revision, Edmonton, 2001. J Rheumatol. 2004; 31:390-2.

2. Ravelli A, Martini A. Juvenile idiopathic arthritis. Lancet. 2007;69(9563):767-78. doi: 10.1016/S0140-6736(07)60363-8.

3. Giancane G, Consolaro A, Lanni S, Davì S, Schiappapietra B, Ravelli A Juvenile Idiopathic Arthritis Diagnosis and Treatment. RheumatolTher. 2016;3(2):187-207.

4. Giancane G, Alongi A, Ravelli A. Update on the pathogenesis and treatment of juvenile idiopathic arthritis. CurrOpinRheumatol. 2017;29(5):523-9. doi: 10.1097/BOR.0000000000000417.

5. Poli C, De la Puente L, Hoyos-Bachiloglu R, Cerda J, Borzutzky A. Pediatric Rheumatology Admissions in Chile, 2001-2010: Unavailability of a Pediatric Rheumatologist May Hinder a Correct Diagnosis. Arthritis \& Rheumatology. 2014;66:S183. doi: 10.1002/art.38561.

6. Horneff G, Klein A, Ganser G, et al. Protocols on classification, monitoring and therapy in children's rheumatology (PRO-KIND): results of the working group Polyarticular juvenile idiopathic arthritis. PediatrRheumatol Online J. 2017;15(1):78. doi: 10.1186/s12969-0170206-9.
7. Klotsche J, Minden K, Thon A, Ganser G, Urban A, Horneff G. Improvement in Health-Related Quality of Life for Children With Juvenile Idiopathic Arthritis After Start of Treatment With Etanercept. Arthritis Care \& Research. 2014;66(2):253-62 DOI 10.1002/ acr.22112.

8. Cimaz R, Marino A, Martini A. How I treat juvenile idiopathic arthritis: A state of the art review. Autoimmun Rev. 2017;16(10):1008-15. doi: 10.1016/j. autrev.2017.07.014.

9. Vanoni F, Minoia F, Malattia C. Biologics in juvenile idiopathic arthritis: a narrative review. Eur J Pediatr. 2017; 176(9):114753. doi: 10.1007/s00431-017-2960-6.

10. Wallace C, Giannini E, Spalding S, et al. Trial of early aggressive therapy in polyarticular juvenile idiopathic arthritis. Arthritis Rheum. 2012; 64(6):2012-21. doi: 10.1002/art.34343.

11. Aird A, Aranguiz P, Barría R, et al. Guía Clínica GES de Artritis Idiopática Juvenil 2014 Rev. Chil. Reumatol. 2014;30(3):98118.

12. Strickler A, Palma J, Charris R, et al. Contribution of the use of basic telemedicine tools to the care of children and adolescents with juvenile idiopathic arthritis at the Puerto Montt Hospital, Chile. Rev ChilPediatr. 2018;89(1):59-66. doi: 10.4067/S0370-41062018000100059.

13. Miranda M, Talesnik E, González B, et al. Enfermedades reumáticas y del tejido conectivo en niños de Santiago, Chile. Rev
ChilPediatr 1996; 67:200-5.

14. Consolaro A, Ravelli A. Unraveling the Phenotypic Variability of Juvenile Idiopathic Arthritis across Races or Geographic Areas--Key to Understanding Etiology and Genetic Factors? J Rheumatol. 2016;43(4):683-5. doi: 10.3899/jrheum.160173.

15. Nordal E, Zak M, Aalto K, et al. Ongoing disease activity and changing categories in a long-term nordic cohort study of juvenile idiopathic arthritis. Arthritis Rheum. 2011;63(9):2809-18. doi: 10.1002/ art.3042.

16. Shoop-Worrall S, Kearsley-Fleet L, Thomson W, Verstappen S, Hyrich K. How common is remission in juvenile idiopathic arthritis: A systematic review Seminars Arthritis Rheum 2017;47(3):331-7. doi: 10.1016/j. semarthrit.2017.05.007.

17. Glerup M, Herlin T, Twilt M. Clinical Outcome and Long-term Remission in JIA. CurrRheumatol Rep. 2017;19(12):75. doi: 10.1007/s11926-017-0702-4.

18. Minden K, Horneff G, Niewerth M, et al. The time of DMARD start in JIA determines the likehood of a drug-free remission in young adulthood. Arthritis Care Res (Hoboken). 2019;71(4):471-81. doi: 10.1002/acr.23709.

19. Wallace C, Giannini E, Spalding S, et al. Clinically inactive disease in a cohort of children with new-onset polyarticular juvenile idiopathic arthritis treated with early aggressive therapy: time 
to achievement, total duration, and predictors. J Rheumatol. 2014;41(6):116370. doi: 10.3899/jrheum. 131503.

20. Albers H, Brinkmann D, Kamphuis S, et al. Clinical course and prognostic value of disease activity in the first two years in different subtypes of juvenile idiopathic arthritis. Arthritis Care Res. 2010;62(2):204-12.

21. Ammerlaan J, Scholtus L, Bijlsma H, Prakken B, Kruize A. An urge for change: transitional care for young adults with juvenile idiopathic arthritis. Patient EducCouns. 2013;92(1):127-9. doi: 10.1016/j.pec.2013.02.006.

22. Stringer E, Scott R, Mosher D, et al. Evaluation of a Rheumatology Transition Clinic. PediatrRheumatol Online J. 2015; 13: 22. doi: 10.1186/s12969-015-0016-x.

23. Mannion ML, Xie F, Baddley J, et al. Analysis of health care claims during the peri-transfer stage of transition from pediatric to adult care among juvenile idiopathic arthritis patients. PediatrRheumatol Online J. 2016;14(1):49. doi: 10.1186/s12969-0160107-3.

24. Cruikshank M, Foster HE, Stewart J, Davidson JE, Rapley T. Transitional care in clinical networks for young people with juvenile idiopathic arthritis: current situation and challenges. ClinRheumatol. 2016;35(4):893-9. doi: 10.1007/s10067015-2950-x.

25. Hilderson D, Westhovens R, Wouters C, Van der Elst K, Goossens E, Moons P. Rationale, design and baseline data of a mixed methods study examining the clinical impact of a brief transition programme for young people with juvenile idiopathic arthritis: the DON'T RETARD project. BMJ Open. 2013;3(12):e003591. doi: 10.1136/ bmjopen-2013-003591.
26. Foster H, Minden K, Clemente D, et al. EULAR/PReS standards and recommendations for the transitional care of young people with juvenileonset rheumatic diseases Ann Rheum Dis. 2016;0:1-8. doi:10.1136/ annrheumdis-2016-210112.

27. Conti F, Pontikaki I, D’Andrea M, Ravelli A, De Benedetti F.Patients with juvenile idiopathic arthritis become adults: the role of transitional care. Clinical and Experimental Rheumatology 2018;36(6):1086-94.

28. Coulson EJ, Hanson HJ, Foster HE. What does an adult rheumatologist need to know about juvenile idiopathic arthritis? Rheumatology (Oxford). 2014;53(12):2155-66. doi: 10.1093/ rheumatology/keu257.

29. Feger D, Longson N, Dodanwala H, Ostrov B, Olsen N, June R. Comparison of Adults with Polyarticular Juvenile Idiopathic Arthritis to Adults with Rheumatoid Arthritis: A Cross-sectional Analysis of Clinical Features and Medication Use. J ClinRheumatol. 2018; 17. doi: $10.1097 /$ RHU.0000000000000819.

30. Hersh A, Prahalad S. Genetics of Juvenile Idiopathic Arthritis. Rheum Dis Clin North Am. 2017;43(3):435-448. doi: 10.1016/j.rdc.2017.04.007.

31. De Silvestri A, Capittini C, Poddighe D, et al. HLA-DRB1 alleles and juvenile idiopathic arthritis: Diagnostic clues emerging from a meta-analysis. Autoimmun Rev 2017;16(12):1230-6. doi.org/10.1016.

32. Jamilloux $Y$, Gerfaud-Valentin M, Martinon F, Belot A, Henry T, Sève P. Pathogenesis of adult-onset Still's disease: new insights from the juvenile counterpart. Immunol Res. 2015;61(12):53-62. doi: 10.1007/s12026014-8561-9.
33. Yang J, Lee E, Seo J, Jung J, Suh C, Kim $\mathrm{H}$. Application of the international league against rheumatism classification criteria for systemic juvenile idiopathic arthritis as a prognostic factor in patients with adultsonset Still's disease. PediatrRheumatol Online J. 2018;16(1):9. doi: 10.1186/ s12969-018-0225-1.

34. Goirand M, Breton S, Chevallier F, et al. Clinical features of children with enthesitis-related juvenile idiopathic arthritis/juvenile spondyloarthritis followed in a French tertiary care pediatric rheumatology centre. Pediatric Rheumatology 2018;16:21. Doi.org 10.1186/s12969-018-0238-9.

35. Martini A. It is time to rethink juvenile idiopathic arthritis classification and nomenclature. Ann Rheum Dis. 2012;71(9):1437-9. doi: 10.1136/ annrheumdis-2012-201388.

36. Nigrovic PA, Raychaudhuri S, Thompson SD. Review: Genetics and the Classification of Arthritis in Adults and Children. Arthritis Rheumatol. 2018;70(1):7-17. doi: 10.1002/art.40350.

37. Martini A. Are the number of joints involved or the presence of psoriasis still useful tools to identify homogeneous disease entities in juvenile idiopathic arthritis? J Rheumatol. 2003;30(9):1900-3.

38. Ritchlin C, Colbert R and Gladman D. Psoriatic Arthritis. N Engl J Med 2017;376:957-70. doi: 10.1056/ NEJMra1505557.

39. Belot A. New classification for juvenile idiopathic arthritis: Is the Tower of Babel falling? Joint Bone Spine. 2018;85(2):13941. doi: 10.1016/j.jbspin.2017.08.001.

40. An Q, Jin MW, An XJ, Xu SM, Wang L. Macrophage activation syndrome as a complication of juvenile rheumatoid arthritis. Eur Rev Med Pharmacol Sci. 2017;21(19):4322-6. 